\title{
Fat-Free Mass Can Be Utilized to Assess Resting Energy Expenditure for Male Athletes of Different Body Size
}

\author{
Satomi OSHIMA $^{1}$, Sakiho MIYAUCHI ${ }^{1}$, Hiroshi KAWANO ${ }^{2}$, \\ Toshimichi IsHIJIMA ${ }^{3}$, Meiko AsAKA ${ }^{1}$, Motoko TAGUCHI ${ }^{4}$, Suguru TorII ${ }^{2}$ \\ and Mitsuru HiguCHI ${ }^{2}$ \\ ${ }^{1}$ Graduate School of Sport Sciences, ${ }^{2}$ Faculty of Sport Sciences, and ${ }^{3}$ Waseda Sport Sciences Research Center, \\ Waseda University, 2-579-15 Mikajima, Tokorozawa, Saitama 359-1192, Japan \\ ${ }^{4} J a p a n$ Women's College of Physical Education, 8-19-1 Kita-Karasuyama, Setagaya-ku, \\ Tokyo 157-8565, Japan \\ (Received March 29, 2011)
}

\begin{abstract}
Summary The fat-free mass (FFM) of athletes is typically large, and thus the FFM is often utilized to estimate their resting energy expenditure (REE). While the proportional contribution of organ-tissues to the total influence of FFM on REE is known for untrained individuals and female athletes, the extent to which this is valid for male athletes is unclear. The purpose of this study was to clarify the contribution of the components of FFM to REE in male athletes. Fifty-seven male athletes participated in this study. REE was assessed by indirect calorimetry and body composition by dual X-ray absorptiometry. The athletes were equally divided into three groups based on FFM: Small (S), Medium (M), and Large (L). When measured REE (REEm) was compared with REE estimated (REEe) based on the four organ-tissue compartments with set metabolic rates, REEm and REEe had a strong association $(r=0.76$, $p<0.001)$. In addition, the absolute value of total REE became larger in accordance with body size (S: 1,643 $\pm 144, \mathrm{M}: 1,865 \pm 140$, and L: $2,060 \pm 156 \mathrm{kcal} / \mathrm{d}$ ) accompanied by increases in mass of all four organ-tissue compartments as body size increased. The consistency of REE/FFM in male athletes in spite of the difference in body size can be explained by the steadiness among the three groups of the relative contribution of each organ-tissue compartment to the FFM. Based on these results, the FFM is the major determinant of REE regardless of body size in male athletes.
\end{abstract}

Key Words resting energy expenditure, male athletes, fat-free mass, organ-tissue mass

Resting energy expenditure (REE) is often used as a basis for estimating an individual's energy requirement (EER). EER can be roughly assessed by multiplying REE by physical activity level (PAL), as noted in Dietary Reference Intakes for Japanese, 2010 (DRIs) (1). Therefore, an accurate way to estimate and predict an athlete's REE would be of great value.

REE is known to be influenced by age, sex, body size, and hormone levels, as well as body composition $(2,3)$. The major determinant of REE is fat-free mass (FFM) (4). Consequently, FFM is routinely used to estimate REE for athletes who have a large FFM to body weight (BW) ratio. A formula established by the Japan Institute of Sports Sciences utilizes REE per kg FFM and is often employed to estimate REE for athletes (5). However, FFM is not an energetically homogeneous compartment, but rather consists of a variety of heat-producing components (6). The metabolic rate of internal organs can differ substantially. For example, liver, brain, heart, and kidney have metabolic rates of 200, 240, 440, and $440 \mathrm{kcal} / \mathrm{kg} / \mathrm{d}$, respectively, as compared to adipose tissue, which is quite low at $4.5 \mathrm{kcal} / \mathrm{kg} / \mathrm{d}$ (6). Even skeletal muscle, which is commonly considered a metaboli-

\footnotetext{
*E-mail: satomioshima@fuji.waseda.jp
}

cally active tissue, has a metabolic rate of only $13 \mathrm{kcal} /$ $\mathrm{kg} / \mathrm{d}$. In order to establish a more accurate estimation of the relationship of FFM to REE, FFM may need to be compartmentalized at the organ-tissue level.

An advance in this regard was made by Heymsfield et al. (7), who evaluated the contribution of organ-tissue mass on REE using a four organ-tissue compartment scheme. The compartments they utilized were bone mass (BM), adipose tissue (AT), skeletal muscle (SM), and residual mass (RM). Their subjects were untrained males and females. They found that REE/FFM decreased as FFM increased, due to the fact that the RM to FFM ratio was not constant, but instead decreased with increases in FFM. Because the RM is a more metabolically active compartment than the others, the REE/FFM would decrease as FFM increased; therefore estimating REE based simply on FFM is inadequate. However, their finding was based on untrained individuals, and it is not clear to what extent their findings would apply to athletic individuals with sport-specific distinct body features. Athletes in general have a body composition characterized by a greater FFM as compared to untrained individuals $(8)$. Therefore, if the REE/FFM is not constant due to the difference in the proportion of organ-tissue mass to FFM, the error between measured 
and calculated REE becomes larger when estimating REE based on FFM. However, there has been little research investigating the relationship between FFM and REE at the organ-tissue level on male athletes, so the details of this relationship remain unclear. For female athletes, Taguchi et al. (9) reported that FFM can predict REE since between-individual differences in REE can be explained by differences in the metabolically active organ-tissue mass. However, for male athletes, the accuracy of an estimation of REE based on FFM has not been studied in detail.

In this study, the purpose was to evaluate the above approach by assessing the relationship between FFM and REE at the organ-tissue level for male athletes. The working hypothesis was that the REE to FFM ratio would remain constant due to the uniformity of contribution of the organ-tissue masses that make the major contribution to FFM. Thus, the FFM would be a valid predictor for determining the REE of male athletes.

\section{METHODS}

Subjects. Fifty-seven healthy collegiate male athletes aged between 18 and $22 \mathrm{y}$ participated in this study. They belonged to either the American football (AF) $(n=42)$ or Handball $(\mathrm{HB})(n=15)$ teams. Both teams were ranked within the top three at the National Collegiate championships in 2010. None of the subjects had a history of cardiovascular, endocrine, or orthopedic disorders nor had any been taking any medication when the measurements were taken. Since thyroid hormone influences metabolic rate (10), the blood level of a thyroid hormone (triiodothyronine; $\mathrm{T}_{3}$ ) was evaluated for each subject using the Chemiluminescence Immunoassay (CLIA) technique. The values ranged from 70 to $176 \mathrm{ng} / \mathrm{dL}$, which is within the normal range. The subjects were placed in one of three groups according to their FFM. The groups all consisted of 19 individuals and were termed Small (S), Medium (M), and Large (L) based on the significant differences in FFM between the groups to represent each body size (Table 1). The compositions of these groups were as follows; $\mathrm{S}: \mathrm{AF}=18$ and $\mathrm{HB}=1$; and both $\mathrm{M}$ and $\mathrm{L}: \mathrm{AF}=12$ and $\mathrm{HB}=7$. Subjects were given a verbal and written description of the study and their informed consent was obtained before testing. The study was approved by the Human Research Ethics Committee of the Faculty of Sport Sciences of Waseda University for use of human subjects in accordance with the Declaration of Helsinki.

Body composition measurements. Body weight (BW) was measured to the nearest $0.1 \mathrm{~kg}$ by using an electronic scale (Inner Scan BC-660, Tanita Co., Tokyo, Japan). Standing height (Ht) with minimal clothing and with the shoes off was measured to the nearest $0.1 \mathrm{~cm}$ by using a stadiometer (YL-65, Yagami Inc., Nagoya, Japan). The body mass index (BMI) was calculated by dividing BW in kilograms by the square of Ht in meters $\left(\mathrm{kg} / \mathrm{m}^{2}\right)$.

Dual-energy X-ray absorptiometry (DXA) (Hologic QDT-4500, DXA Scanner, Hologic Inc., Whaltham, MA, USA), a relatively easy and non-invasive technique
(11), was used to measure bone mineral content (BMC) (g), lean soft tissue mass ( $\mathrm{g}$ ), and \% body fat. FFM and fat mass (FM) were then calculated based on BW and \% body fat. Subjects wore loose-fitting light cloth without any metal objects and were positioned supine on the scanning table to perform the total body scan.

REE measurement. REE was measured by open-circuit indirect calorimetry using a Douglas bag. Subjects came to the testing facility in the early morning. In the $12 \mathrm{~h}$ period before testing, no food or liquid (other than water) was consumed (12). The subjects were asked to minimize any exertion prior to the laboratory visit for REE determination. After a 30-40 min period of rest in the supine position with the mask on (Rudolph mask; Hans Rudolph Inc., Kansas City, MO, USA), two 10-min samples of expired gas were collected in the bags. Resting heart rate and body temperature were measured during the rest period to confirm an adequate duration of the rest period. The laboratory was kept at a neutral temperature $\left(20-25^{\circ} \mathrm{C}\right)$ based on the previous report (12), and noise was kept to a minimum. The subjects were instructed to remain awake, quiet, and motionless before and throughout the measuring periods.

Oxygen and carbon dioxide concentrations were analyzed using an expiration gas analyzer (Minato AE300S; Minato Medical Science, Tokyo, Japan). The volume of expired air was determined by a dry gas volume meter (DC-5; Shinagawa Corp., Japan) and converted to a standard temperature, pressure, and dry condition (STPD). Gas exchange results were converted to REE (kcal/d) using Weir's equation (13). The mean of the two measured values was used for analysis.

Organ tissue mass and estimated REE. The masses of the four organ-tissue compartments were estimated based on values obtained from dual energy X-ray absorptiometry (DXA) using the previously reported prediction model (7) as follows: BM was calculated by multiplying BMC times 1.85. FM was assumed to be $85 \%$ of total body AT, therefore AT could be calculated by multiplying FM times 1.18. SM was estimated using the sum of appendicular lean soft tissues (ALST) and age in a prediction model established by Kim et al. (14). RM was obtained by subtracting the sum of the calculated BM, AT, and SM from BW, and it included internal organs (i.e. heart, brain, liver, kidneys, spleen, and gastrointestinal tract) as well as skin and glands.

$$
\begin{aligned}
\mathrm{BM}(\mathrm{kg})= & \mathrm{BMC}(\mathrm{g}) \times 1.85 / 1,000 \\
\mathrm{AT}(\mathrm{kg})= & \mathrm{FM}(\mathrm{kg}) \times 1.18 \\
\mathrm{SM}(\mathrm{kg})= & 1.13 \times \mathrm{ALST}(\mathrm{kg})-0.02 \times \text { age }(\mathrm{y})+ \\
& (0.61 \times \mathrm{sex}: \text { male }=1)+0.97 \\
\mathrm{RM}(\mathrm{kg})= & \mathrm{BW}-(\mathrm{BM}+\mathrm{AT}+\mathrm{SM})
\end{aligned}
$$

Values for the resting metabolic rate of each of the four compartments was adopted from values published in a previous study: $2.3 \mathrm{kcal} / \mathrm{kg}$ for BM, $4.5 \mathrm{kcal} / \mathrm{kg}$ for AT, $13 \mathrm{kcal} / \mathrm{kg}$ for SM, and $54 \mathrm{kcal} / \mathrm{kg}$ for RM (6). Estimated REE (REEe) was obtained based on the sum of the four body compartments (BM, AT, SM, and RM) by multiplying the corresponding tissue respiration rates as follows:

$\mathrm{REEe}=2.3 \mathrm{BM}+4.5 \mathrm{AT}+13 \mathrm{SM}+54 \mathrm{RM}$ 
Table 1. Subjects characteristics.

\begin{tabular}{|c|c|c|c|c|}
\hline & All & Small & Medium & Large \\
\hline & $(n=57)$ & $(n=19)$ & $(n=19)$ & $(n=19)$ \\
\hline Age (y) & $19.7 \pm 1.2$ & $18.8 \pm 1.0^{*}$ & $19.7 \pm 0.8$ & $20.5 \pm 1.1^{\dagger}$ \\
\hline Height (cm) & $175.3 \pm 6.4$ & $171.2 \pm 5.9$ & $174.8 \pm 6.0$ & $180.0 \pm 4.1^{* \dagger}$ \\
\hline BW (kg) & $78.4 \pm 11.5$ & $67.1 \pm 4.8^{*}$ & $77.1 \pm 2.9$ & $90.9 \pm 8.8^{* \dagger}$ \\
\hline BMI $\left(\mathrm{kg} / \mathrm{m}^{2}\right)$ & $25.5 \pm 3.2$ & $22.9 \pm 1.5^{*}$ & $25.3 \pm 2.0$ & $28.1 \pm 3.4^{* \dagger}$ \\
\hline Body fat (\%) & $14.2 \pm 3.9$ & $12.9 \pm 2.4$ & $13.1 \pm 3.5$ & $16.5 \pm 4.6^{\dagger}$ \\
\hline FM (kg) & $11.3 \pm 4.7$ & $8.7 \pm 1.9$ & $10.2 \pm 2.9$ & $15.0 \pm 5.8^{* \dagger}$ \\
\hline FFM (kg) & $67.0 \pm 8.1$ & $58.4 \pm 4.1^{*}$ & $66.7 \pm 1.9$ & $75.9 \pm 4.6^{* \dagger}$ \\
\hline
\end{tabular}

Values are mean \pm SD.

BW: body weight, BMI: body mass index, FM: fat mass, FFM: fat-free mass.

Significance was determined by one-way analysis of variance (one-way ANOVA).

* Significantly different vs Medium $(p<0.05)$.

† Significantly different vs Small $(p<0.05)$.

Table 2. Measured (REEm) and estimated resting energy expenditure (REEe).

\begin{tabular}{|c|c|c|c|c|}
\hline & All & Small & Medium & Large \\
\hline & $(n=57)$ & $(n=19)$ & $(n=19)$ & $(n=19)$ \\
\hline \multicolumn{5}{|l|}{ REEm } \\
\hline (kcal/d) & $1,856 \pm 225$ & $1,643 \pm 144^{*}$ & $1,865 \pm 140$ & $2,060 \pm 156^{* \dagger}$ \\
\hline (kcal/kg BW/d) & $23.8 \pm 1.8$ & $24.5 \pm 1.4$ & $24.2 \pm 1.8$ & $22.8 \pm 1.7^{\dagger}$ \\
\hline$(\mathrm{kcal} / \mathrm{kg} \mathrm{FFM} / \mathrm{d})$ & $27.8 \pm 1.9$ & $28.1 \pm 1.6$ & $28.0 \pm 2.3$ & $27.2 \pm 1.7$ \\
\hline \multicolumn{5}{|l|}{ REEe } \\
\hline$(\mathrm{kcal} / \mathrm{d})$ & $1,848 \pm 229$ & $1,614 \pm 121^{*}$ & $1,855 \pm 97$ & $2,076 \pm 164^{* \dagger}$ \\
\hline
\end{tabular}

Values are mean \pm SD.

BW: body weight, FM: fat mass, FFM: fat-free mass.

Significance was determined by one-way analysis of variance (one-way ANOVA).

* Significantly different vs Medium $(p<0.05)$.

$\dagger$ Significantly different vs Small $(p<0.05)$.

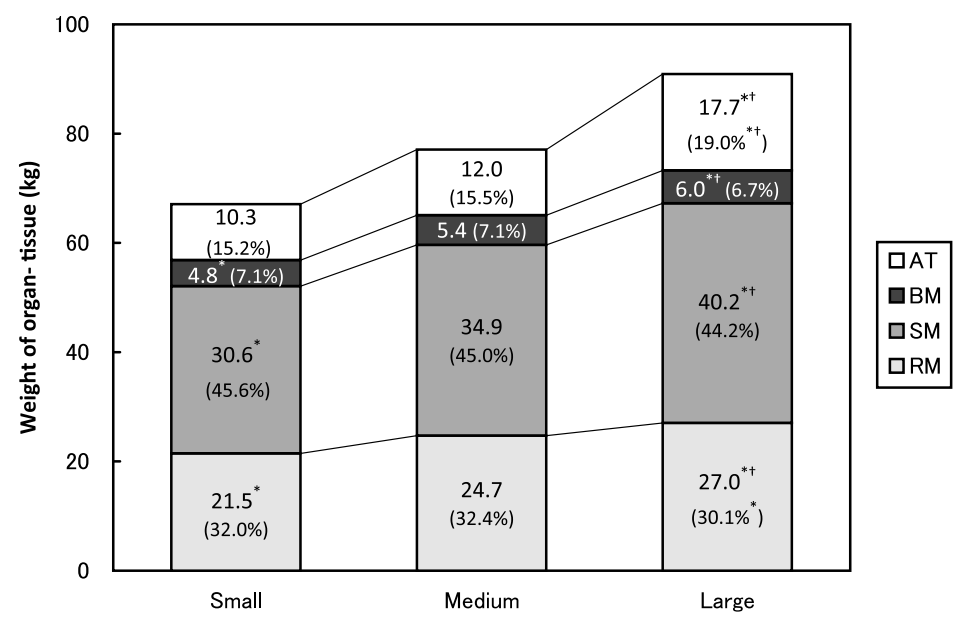

Fig. 1. Four organ tissue components expressed as a weight and their respective fractional contribution to BW. BM: bone mass, AT: adipose tissue, SM: skeletal muscle, RM: residual mass. Significance was determined by one-way ANOVA. * Significantly different vs M $(p<0.05),{ }^{\dagger}$ Significantly different vs S $(p<0.05)$.

Statistical analysis. The data were expressed as mean \pm standard deviation (SD) for all variables. SPSS ver. 17.0 was used for statistical analysis (SPSS Inc., Chicago, IL, USA). The differences among the three body size groups were analyzed utilizing a one-way analysis of variance (ANOVA). The Tukey test was employed to locate the source of the significant differences where appropriate. The level of significance was defined as $p<0.05$ for all statistical analyses. 


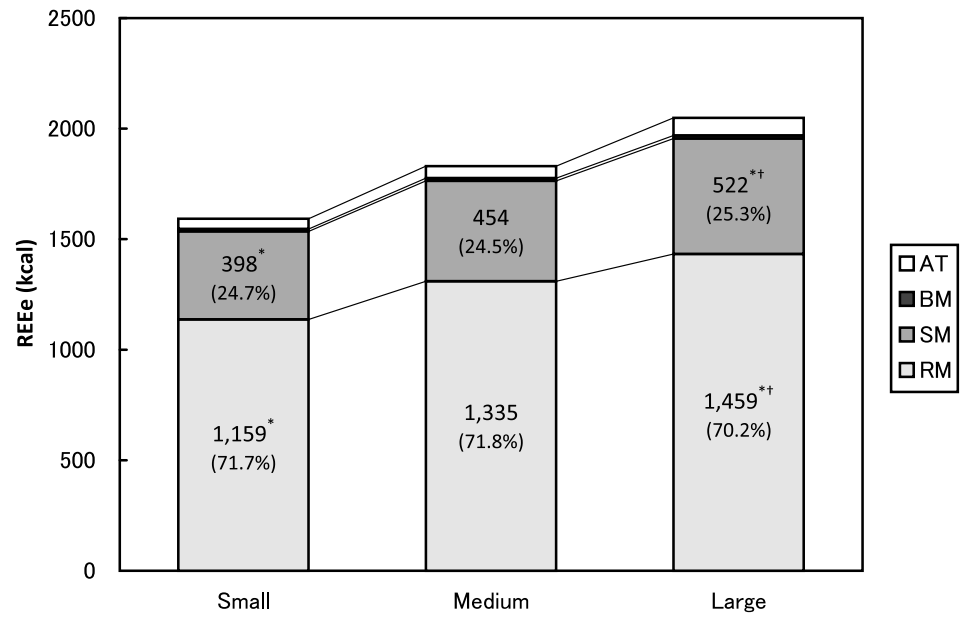

Fig. 2. Four organ-tissue components expressed as specific energy expenditure. BM: bone mass, AT: adipose tissue, SM: skeletal muscle, RM: residual mass. Significance was determined by one-way ANOVA. *Significantly different vs M $(p<0.05),{ }^{\dagger}$ Significantly different vs $S(p<0.05)$.

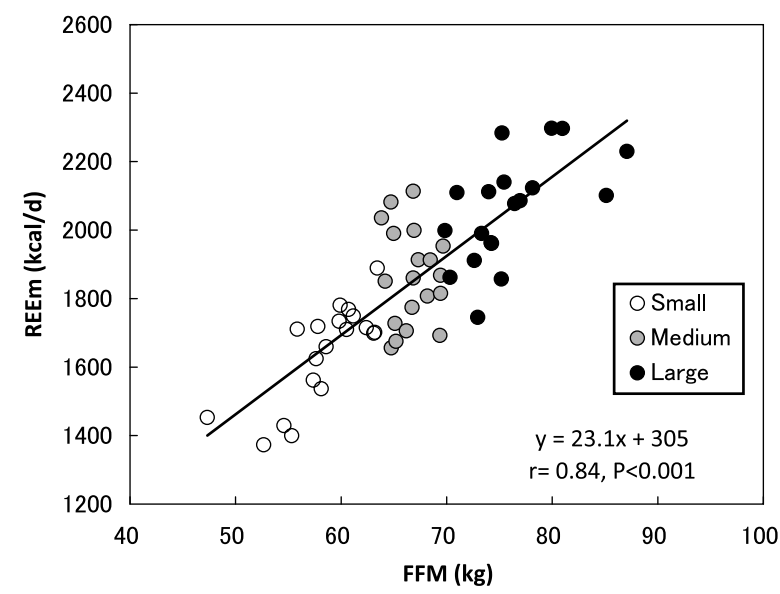

Fig. 3. Relationship between fat-free mass and measured REE.

\section{RESULTS}

\section{Subjects' characteristics}

The characteristics of the subjects are shown in Table 1. The mean age of each body size group tended to increase with increases in body size. Ht was significantly greater in the Large group. BW and BMI significantly increased as body size increased. FM and \% body fat were significantly higher in the Large group.

Measured and estimated REE

The overall average of absolute REE increased significantly in accordance with FFM (Table 2 and Fig. 2). Only the Large group showed a significantly lower REE/ BW ratio when compared to the other two body size groups (Table 2). However, when REE was divided by FFM, there were no longer significant differences among the three groups. REEm and REEe showed a significant correlation $(r=0.76, p>0.001)$, and the difference between REEm and REEe was $8 \pm 158 \mathrm{kcal}$.

FFM contribution to REE

The absolute and relative values of each of the four organ-tissue compartments are shown in Fig. 1. The masses of BM, SM, and RM increased significantly in

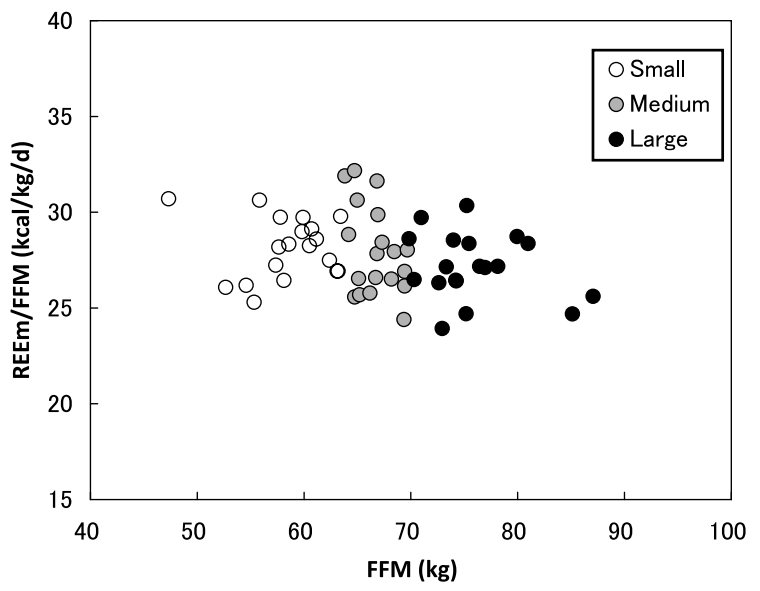

Fig. 4. Relationship between fat-free mass and measured REE/FFM.

accordance with larger body size. The average mass of AT was found to be significantly larger in the Large group as compared to the masses of AT in the other two groups. Relative masses of BM and SM were relatively similar among the three groups. On the other hand, AT and RM were higher in the Large group as compared to the Small and Medium groups for AT, and the Medium group for RM. Figure 2 shows the energy expenditures in $\mathrm{kcal} / \mathrm{d}$ of these four organ-tissue compartments. The relative contributions of SM and RM to REE were not significantly different among the groups. Figures 3 and 4 show the relationship between FFM with REEm and REEm/FFM. REEm was highly correlated with FFM $(r=0.84, p<0.001)$. However, REEm/FFM did not show any correlation with FFM. The contribution percentages of FFM organ-tissue to FFM are shown in Fig. 5. There were no significant differences in relative contribution of each FFM organ-tissue compartment among the three groups.

\section{DISCUSSION}

In the present study, male athletes with a wide range of FFM were utilized to understand the relationship 


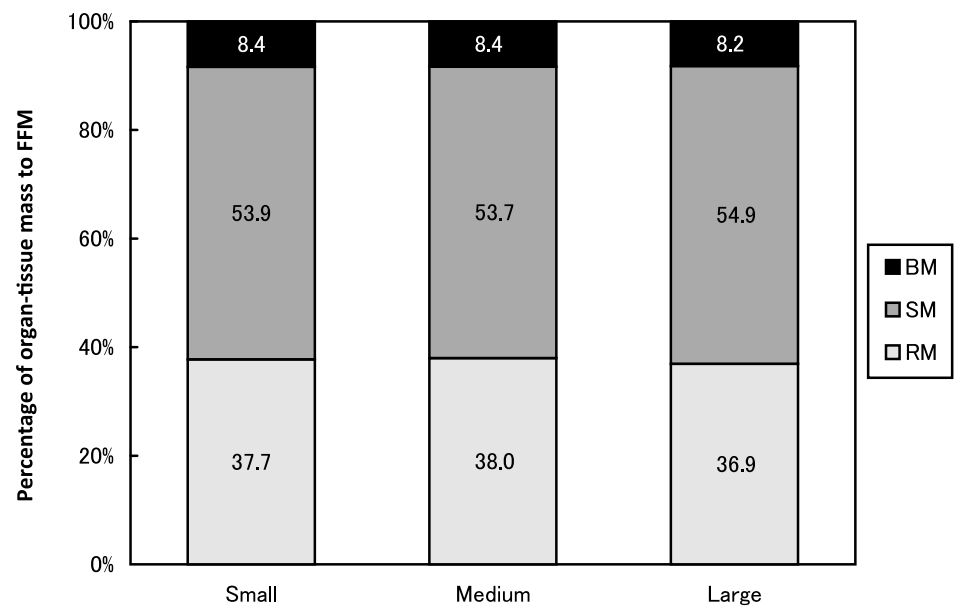

Fig. 5. Percentage contribution of organ-tissue mass to FFM.

between FFM and REE at the organ-tissue level. It was found that, for male athletes, REE/FFM does not change due to the fact that the percentage of organ-tissue mass contribution to FFM is consistent regardless of body size.

It is well known that the REE is attributed to the mass of organ-tissue $(7,15)$. While an approach to the subject has been previously explored at this level, $(4,7,16$, 17), such studies were largely done on untrained individuals. Therefore, it was not known that the same findings would apply to athletic population. Athletes typically train daily, and have a body composition which is different from the norm and is characterized by a large FFM (8). Therefore, there may be different, relatively unique factors for athletes that might influence the value of REE. In fact, Grund et al. (18) found that resistance-trained men had a higher REE compared to untrained men even after REE was evaluated as a ratio by dividing it by FFM. However, the explanation for this finding has yet to be uncovered. Taguchi et al. (9) reported that organ-tissue mass is responsible for determining REE, and thus it can be accurately measured by FFM. The investigator utilized Japanese female athletes over a wide range of body sizes. However, a number of factors that might influence REE, such as average FFM and \% body fat, as well as sex hormones (19) differ between males and females. These are the reasons why the investigation of REE in relation to FFM must be done separately for athletes and untrained individuals, and also for males and females.

In the present study, we used four compartments constituting a whole body to see the relationship of organ-tissue mass with REE for male athletes (Fig. 1). It was found that all four compartments except for AT between Small and Medium groups, increased as body size increased. The four organ-tissue compartments have a specific metabolic rate; BM and AT have relatively smaller weight-related metabolic rates $(2.3$ and $4.5 \mathrm{kcal} / \mathrm{kg} / \mathrm{d}$ ), on the other hand, SM and RM have higher metabolic rates (13 and $54 \mathrm{kcal} / \mathrm{kg} / \mathrm{d}$ ) (7). REEe can be obtained utilizing these metabolic rates with the actual mass of each organ-tissue compartment based on the previously established model $(9,11)$. According to previous studies which used the same organ-tissue REE prediction method, the correlation coefficients between REEm and REEe were $0.75(p<0.001)$ for untrained males and females, and $0.77(p<0.01)$ for Japanese female athletes $(7,9)$. On the other hand, the correlation coefficient was $0.76(p<0.001)$ in the present study. The estimation error in a study by Usui et al. (11) using the same methodology was $19 \pm 105 \mathrm{kcal}$ in the group of young high-fitness females, in comparison with $8 \pm 158 \mathrm{kcal}$ in the present study. Based on these reports, the organ-tissue prediction model is a well established method to predict REE and we assumed REE was adequately predicted by using the same method. Therefore, we hold that for male athletes differences in the dependence of REE on body size can be attributed to changes in organ-tissue mass, just like that of untrained individuals and female athletes. RM includes different internal organs such as the brain, heart, liver, and kidneys (240, 440, 200, and $440 \mathrm{kcal} /$ $\mathrm{kg} / \mathrm{d}$ ) and these organs have exceptionally high weightrelated metabolic rates when compared to the tissues of other organs of the body (6). Thus, RM has highest metabolic rates among the four organ-tissue compartments ( $54 \mathrm{kcal} / \mathrm{kg} / \mathrm{d})$. In fact, RM alone contributes $71 \%$ of the total REE (Fig. 4). A previous study with Sumo wrestlers has shown that the FFM contribution to the sum of four organs (brain, heart, liver, and kidney) was $6.3 \%$ in comparison with $6.6 \%$ for a control group; thus the difference was not prominently large. Therefore, it is reasonable to assume that a metabolic rate of $54 \mathrm{kcal} / \mathrm{kg}$ for RM can also apply to the athletes of the present study. On the other hand, although, the metabolic rate of SM is much smaller than RM (SM: $13 \mathrm{kcal} /$ $\mathrm{kg} / \mathrm{d}$ vs RM: $54 \mathrm{kcal} / \mathrm{kg} / \mathrm{d}$ ), athletes have large absolute mass as in SM. As a result, SM accounts for about 25\% of REE, and together, SM and RM account for about 95\% of the total REE. As for AT, it increases not just as absolute mass, but also as \% AT and \% body fat in accordance with increase in body size (Fig. 3). For this reason, it suggests that athletes with larger body size in regards to FFM tend to have more FM. However, since 
AT has a relatively low metabolic rate $(4.5 \mathrm{kcal} / \mathrm{kg} / \mathrm{d})$, it has little influence on total REE (3.2\% of REE). BM also increases as body size increases without the change in the percentage contribution to BW between the groups (Fig. 1), but it accounts for less than 1\% of REE (Fig. 2).

In consequence, FFM is largely determined by SM and RM and is thus the major determinant of REE. In the present study, we found that REEm was highly associated with FFM, which agrees with previous studies (6, 7, 9) (Fig. 3). FFM consists of metabolically high compartments such as SM and RM; hence REE correlates with FFM. On the other hand, REE/FFM did not change in accordance with FFM (Fig. 4). This finding is at variance with the results of Heymsfield et al. (7) which indicate that REE/FFM decreases as FFM increases. They based their finding on the decrease of the contribution of RM to FFM as FFM increases. According to Midorikawa et al. (20), the REE/FFM ratio of both controls and Sumo wrestlers were the same, even though the difference between the two groups was more than $40 \mathrm{~kg}$ in BW and $25 \mathrm{~kg}$ in FFM. This result implies that organ-tissue mass may increase in parallel with FFM, and as expected, it was actually the case that the proportion of BM, SM, and RM to FFM were similar between the groups (Fig. 5). Therefore, the reason that REE/FFM for athletes was consistent regardless of FFM may be the steadiness of the organ-tissue contribution to FFM. Furthermore, according to Illner et al. (21), even though muscle and organs are constituents of FFM, when compared the correlation coefficients of FFM, muscle mass, and sum of organs with REE, FFM was most associated with REE. If these results are upheld, then FFM is a reliable variable to use when estimating REE for male athletes of all sizes.

There are two possible limitations to this study. First, the metabolic rates of each organ-tissue could not be directly measured and was assumed to be constant based on the results of previous studies $(7,11,22)$. A second limitation is the subjects were recruited from a limited set of sports. In order to generalize the findings from this study to all Japanese male athletes, the utilization of subjects from a broader range of sports would be necessary. For these reasons, further study may be necessary to confirm the findings from the present study by assessing athletes from variety of sports types including endurance athletes with relatively a smaller FFM.

In conclusion, differences in REE in accordance with body size in male athletes were accounted for by observed differences in organ-tissue mass, especially SM and RM. The proportion of the body organ-tissue compartment was found to be consistent over the utilized range of FFM. This resulted in REE/FFM remaining constant; therefore it is appropriate for FFM to be considered the major contributor when determining REE for male athletes.

\section{Acknowledgments}

We would like to sincerely thank the volunteer subjects who took part in this study. This study was supported by a research grant for Waseda University Global
COE program "Sports Sciences for Promotion of Active Life.”

\section{REFERENCES}

1) Ministry of Health, Labour and Welfare. 2010. Dietary Reference Intakes for Japanese, p 43-61. Daiichi Shuppan Publishing Co., Ltd., Tokyo.

2) Cunningham JJ. 1980. A reanalysis of the factors influencing basal metabolic rate in normal adults. Am J Clin Nutr 33: 2372-2374.

3) Harris JA, Benedict FG. 1918. A biometric study of human basal metabolism. Proc Natl Acad Sci USA 4: 370-373.

4) Cunningham JJ. 1991. Body composition as a determinant of energy expenditure: a synthetic review and a proposed general prediction equation. Am J Clin Nutr 54: 963-969.

5) Koshimizu T, Yanagisawa K, Higuchi M. 2005. Estimating energy requirement for athletes. J Training Sci Exerc Sport 17: 245-250.

6) Elia M. 1992. Organ and tissue contribution to metabolic rate. In: Energy Metabolism, Tissue Determinants and Cellular Corollaries (Kinney JM, Tucker HK, eds), p 61-80. Raven Press, New York.

7) Heymsfield SB, Gallagher D, Kotler DP, Wang Z, Allison DB, Heshka S. 2002. Body-size dependence of resting energy expenditure can be attributed to nonenergetic homogeneity of fat-free mass. Am J Physiol Endocrinol Metab 282: E132-E138.

8) Japan Olympic Committee. The physical fitness test report of Japan National Team; the 18th Olympic Winter Games (1998/Nagano) the 27th Olympic Summer Games (2000/Sydney).

9) Taguchi M, Takata KI, Tatsuta W, Katsuragi C, Usui C, Sakamoto S, Higuchi M. 2011. Resting energy expenditure can be assessed by fat-free mass in female athletes regardless of body size. J Nutr Sci Vitaminol 57: 22-29.

10) Al-Adsani H, Hoffer LJ, Silva JE. 1997. Resting energy expenditure is sensitive to small dose changes in patients on chronic thyroid hormone replacement. $J$ Clin Endocrinol Metab 82: 1118-1125.

11) Usui C, Takahashi E, Gando Y, Sanada K, Oka J, Miyachi M, Tabata I, Higuchi M. 2009. Resting energy expenditure can be assessed by dual-energy X-ray absorptiometry in women regardless of age and fitness. Eur J Clin Nutr 63: 529-535.

12) Compher C, Frankenfield D, Keim N, Roth-Yousey L. 2006. Best practice methods to apply to measurement of resting metabolic rate in adults: A systematic review. J Am Diet Assoc 106: 881-903.

13) Weir JBDV. 1949. New methods for calculating metabolic rate with special reference to protein metabolism. J Physiol 109: 1-9.

14) Kim J, Wang Z, Heymsfield SB, Baumgartner RN, Gallagher D. 2002. Total-body skeletal muscle mass: Estimation by a new dual-energy X-ray absorptiometry method. Am J Clin Nutr 76: 378-383.

15) Hayes M, Chustek M, Wang Z, Gallagher D, Heshka S, Spungen A, Bauman W, Heymsfield SB. 2002. Dxa: Potential for creating a metabolic map of organ-tissue resting energy expenditure components. Obes Res 10: 969-977.

16) Bosy-Westphal A, Reinecke U, Schlorke T, Illner K, Kutzner D, Heller M, Muller MJ. 2004. Effect of organ and tissue masses on resting energy expenditure in 
underweight, normal weight and obese adults. Int J Obes 28: $72-79$.

17) Muller MJ, Illner K, Bosy-Westphal A, Brinkmann G, Heller M. 2001. Regional lean body mass and resting energy expenditure in non-obese adults. Eur J Nutr 40: 93-97.

18) Grund A, Krause H, Kraus M, Siewers M, Rieckert H, Muller MJ. 2001. Association between different attributes of physical activity and fat mass in untrained, endurance- and resistance-trained men. Eur J Appl Physiol 84: 310-320.

19) Day DS, Gozansky WS, Van Pelt RE, Schwartz RS, Kohrt WM. 2005. Sex hormone suppression reduces resting energy expenditure and beta-adrenergic support of rest- ing energy expenditure. I Clin Endocrinol Metab 90: 3312-3317.

20) Midorikawa T, Kondo M, Beekley MD, Koizum K, Abe T. 2007. High REE in sumo wrestlers attributed to large organ-tissue mass. Med Sci Sports Exerc 39: 688-693.

21) Illner K, Brinkmann G, Heller M, Bosy-Westphal A, Muller MJ. 2000. Metabolically active components of fat free mass and resting energy expenditure in nonobese adults. Am J Physiol Endocrinol Metab 278: E308-E315.

22) Midorikawa T, Sekiguchi O, Beekley MD, Bemben MG, Abe T. 2007. A comparison of organ-tissue level body composition between college-age male athletes and nonathletes. Int J Sports Med 28: 100-105. 